EPJ Web of Conferences 70, 00044 (2014)

DOI: 10.1051/epjconf/ 20147000044

C Owned by the authors, published by EDP Sciences, 2014

\title{
Present and future strategies for Neutrinoless Double Beta de- cay searches
}

\author{
Marco Vignati ${ }^{1, a}$ \\ ${ }^{1}$ INFN Sezione di Roma, P.le A. Moro 2, I-00185 Roma, Italy
}

\begin{abstract}
The study of neutrino properties is one of the fundamental challenges in particle physics. In the last years, several progresses have been made, but still we do not know the absolute mass of neutrinos or whether they are Dirac or Majorana particles. The only way to probe the neutrino nature is through the observation of Neutrinoless Double Beta Decay, a not yet observed nuclear transition which emits two electrons and no neutrinos. In this paper, after a brief introduction to the topic, the present and future strategies to detect this process are presented.
\end{abstract}

\section{Introduction}

Neutrino oscillation experiments have provided indirect evidence for finite neutrino masses. They are sensitive to the squared mass differences between the neutrino species measured, but cannot tell us the absolute mass scale. The importance of neutrino masses is also related to the question of the nature of neutrino. Neutrinos, in fact, are the only fermions for which the Majorana formulation is possible, but this description is indistinguishable from the Dirac one in the limit of vanishing masses.

Until the discovery of the massive nature of neutrinos little attention was dedicated to the issue of Majorana neutrinos. The situation has changed after 1998 and there is a common consensus that the Majorana's theory is indeed the best description for the physical neutrinos. From an experimental point of view, neutrinoless double beta decay is the only accessible process able to test the neutrino nature, and could also give information on the mass scale.

\section{Double beta decay}

The Double Beta Decay (DBD) is a rare spontaneous nuclear transition [1], in which a nucleus (A,Z) decays to another nucleus $(\mathrm{A}, \mathrm{Z}+2)$ with the simultaneous emission of two electrons. Both the parent and the daughter nuclei should be more bound than the intermediate one, in order to avoid the occurrence of the equivalent sequence of two single beta decays. Such a condition, due to the pairing term, is fulfilled in nature by only 35 even-even nuclei. The decay can then proceed both to the ground state or to the first excited states of the daughter nucleus, and in the latter case it is accompanied by the emission of $\gamma$ 's. Being a second-order process of the weak interactions, the DBD has a very low probability which leads to extraordinary long lifetimes, of the order of $10^{20} \mathrm{y}$ or greater. Transitions

\footnotetext{
ae-mail: marco.vignati@roma1.infn.it
} 
accompanied by positron emission or electron capture are also possible but they are usually characterized by lower transition energies and poorer experimental sensitivities and therefore they will not discussed here.

There are two double decay modes: $2 v \mathrm{DBD}$ and $0 v \mathrm{DBD}$. The $2 v \mathrm{DBD}$ mode [1]

$$
(A, Z) \rightarrow(A, Z+2)+2 e^{-}+2 \bar{v}
$$

is allowed by the Standard Model of electro-weak interactions and satisfies the total lepton number conservation. The $0 v \mathrm{DBD}$ mode [2]

$$
(A, Z) \rightarrow(A, Z+2)+2 e^{-}
$$

on the other hand violates the lepton number by two units and is not allowed by the Standard Model. The transition can be represented with the exchange of a virtual neutrino between the two beta vertexes. However the neutrino can be emitted and absorbed at the same time by beta vertexes only if it is equal to its own antiparticle, thus following the theory of E. Majorana [3]. Whitin this framework, the half-life of the $0 v \mathrm{DBD}$ nucleus can be expressed as:

$$
\left[T_{1 / 2}^{0 v}\right]^{-1}=G^{0 v}\left|M^{0 v}\right|^{2}\left|m_{\beta \beta}\right|^{2}
$$

where $G^{0 v}$ is the phase space factor, $\left|M^{0 v}\right|^{2}$ is the nuclear matrix element and $m_{\beta \beta}$ is a linear combination of the neutrino masses $m_{k}$ :

$$
m_{\beta \beta} \equiv \sum_{k=1}^{3}\left|U_{e k}\right|^{2} m_{k} e^{i \phi_{k}} .
$$

In the above equation $U_{e k}$ are the elements of the neutrino mixing matrix and $\phi_{k}$ are the Majorana phases, which presence could lead to cancellations. For example, in the case of a Dirac neutrino, the cancellations are complete since it is equivalent to two degenerate Majorana neutrinos with opposite phases.

An observation of the $0 v \mathrm{DBD}$ would imply that the total lepton number is not conserved and that the neutrino is a Majorana particle, a breakthrough in our understanding of nature. The parameter $m_{\beta \beta}$ is the physical quantity behind the decay rate of different nuclei, which have different phase space factor and nuclear matrix elements. $m_{\beta \beta}$ can be expressed in terms of the presently known and unknown quantities in neutrino physics [4] (Fig. 1): absolute mass scale, represented by the mass of the lightest neutrino $m_{\min }$, the two Majorana phases, and the ordering of the neutrino mass eigenstates. Given the actual knowledge the following scenarios are possible: normal hierarchy, where $m_{1}<m_{2} \ll m_{3}$, and inverted hierarchy, where $m_{3} \ll m_{1}<m_{2}$.

Since the phase space of the transition $\left(G^{0 v}\right)$ goes as $Q^{5}$, isotopes with high Q-values are preferred. High Q-values are preferred also from the experimental point of view, because the background from natural radioactivity decreases with energy. Considering also the abundance in nature, the isotopes of experimental interest are limited to ${ }^{130} \mathrm{Te},{ }^{116} \mathrm{Cd},{ }^{76} \mathrm{Ge},{ }^{136} \mathrm{Xe},{ }^{82} \mathrm{Se},{ }^{100} \mathrm{Mo},{ }^{150} \mathrm{Nd}$ and ${ }^{48} \mathrm{Ca}$ (Tab. 1).

Experiments estimate from data the half-life of the decay. To extract the physical parameter $m_{\beta \beta}$ from Eq. 3 one needs $G^{0 v}$ and $M^{0 v}$. While $G^{0 v}$ can be calculated precisely, the nuclear matrix elements $M^{0 v}$ are difficult to estimate. The discussion of the models and the calculation methods are out of the scope of this paper, for more details refer to Ref. [5]. We recall that estimates from different models differ by a factor around 2 for the same isotope, and that the isotopes listed above have matrix elements of the same order of magnitude. The uncertainty on the matrix elements generate problems when comparing the $m_{\beta \beta}$ values of experiments running different isotopes. 


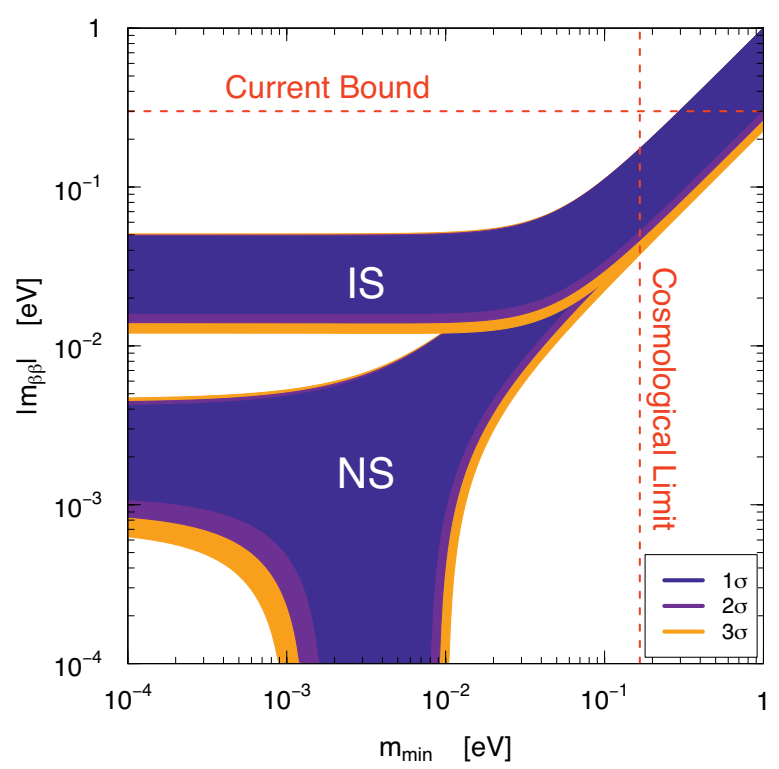

Figure 1. Majorana mass of the neutrino $m_{\beta \beta}$ as a function of the unknown value of the lightest neutrino mass $m_{\min }$. Depending on the ordering of the neutrino mass eigenstates, two patterns are possible: the normal and inverted hierarchy regions. The width of the bands is dominated by the complete ignorance of the Majorana phases, and it is slightly influenced by the uncertainty on the experimental inputs. Picture taken from [4].

\section{Double Beta Decay in experiments}

The signature of the $0 v \mathrm{DBD}$ is a peak in the sum energy spectrum of the electrons at the Q-value of the decay. The sensitivity of an experiment is usually expressed as the process half-life corresponding to the maximum signal that could be hidden by a background fluctuation at a given statistical Confidence Level (CL). Considering the signal as a Gaussian peak over a flat background, the 1- $\sigma$ sensitivity is:

$$
S_{0 v}=\ln 2 \frac{x \eta \epsilon N_{A}}{A} \sqrt{\frac{M T}{B \Delta E}} \quad(68 \% \mathrm{CL})
$$

where $B$ is the background level per detector mass and energy, $M$ is the detector mass, $T$ is the measure time, $\Delta$ is the FWHM energy resolution, $\eta$ is the isotopic abundance of the $\beta \beta$-decaying nucleus, $N_{A}$ the Avogadro number, $A$ the compound molecular mass, $x$ the number of $\beta \beta$ atoms per molecule, and $\epsilon$ the detection efficiency. The desirable features of an experiment are therefore:

- Low background, which requires underground detector operation to shield cosmic rays, very radiopure materials (natural radioactivity decays have typical lifetimes of the order $10^{9-10}$ y versus 
Table 1. Phase space factor $G^{0 v}$, Q-value, natural abundance $a$, and half-life of the 2 neutrino mode of the $0 v$ DBD isotopes of experimental interest. Table taken from Ref. [6], except for the Q.-value of ${ }^{130} \mathrm{Te}$ that was averaged from [7-9].

\begin{tabular}{lccccl} 
isotope & $\begin{array}{c}G^{0 v} \\
{\left[\frac{10^{-14}}{y}\right]}\end{array}$ & $\begin{array}{c}Q_{\beta \beta} \\
{[\mathrm{keV}]}\end{array}$ & $\begin{array}{c}\text { nat. abund. } \\
{[\%]}\end{array}$ & $\begin{array}{c}T_{1 / 2}^{2 v} \\
{\left[10^{20} \mathrm{y}\right]}\end{array}$ & experiments \\
\hline${ }^{48} \mathrm{Ca}$ & 6.3 & 4273.7 & 0.187 & 0.44 & CANDLES \\
${ }^{76} \mathrm{Ge}$ & 0.63 & 2039.1 & 7.8 & 15 & GERDA, Majorana Demonstrator \\
${ }^{82} \mathrm{Se}$ & 2.7 & 2995.5 & 9.2 & 0.92 & SuperNEMO, Lucifer \\
${ }^{100} \mathrm{Mo}$ & 4.4 & 3035.0 & 9.6 & 0.07 & MOON, AMoRe, Lucifer \\
${ }^{116} \mathrm{Cd}$ & 4.6 & 2809 & 7.6 & 0.29 & Cobra \\
${ }^{130} \mathrm{Te}$ & 4.1 & 2528 & 34.5 & 9.1 & CUORE \\
${ }^{136} \mathrm{Xe}$ & 4.3 & 2461.9 & 8.9 & 21 & EXO, Kamland-Zen, NEXT, XMASS \\
${ }^{150} \mathrm{Nd}$ & 19.2 & 3367.3 & 5.6 & 0.08 & SNO+, DCBA/MTD
\end{tabular}

lifetimes longer than $10^{25}$ y for $0 v$ DBD decay) and well-designed passive and/or active shielding against local environmental radioactivity.

- Large source, in order to monitor many candidates nuclei. Present sources contain 10-100 kg of $\beta \beta$ isotope in the most sensitive detectors, while the next generation experiments aim at sources in the $100-1000 \mathrm{~kg}$ scale.

- High energy resolution.

These features cannot be obtained at once with a single detector. Over the years many idea came up to find the optimal technology for a $0 v \mathrm{DBD}$ experiment. The current or next experiments will explore part of the inverted hierarchy of neutrino masses. If the neutrinoless double beta decay will not be observed, an experiment with 1 ton of isotope and a background less than 1 count/ton/y below the peak will be required to cover entirely the inverted hierarchy region. In the following we review status and prospects of the leading techniques in the field, hoping that the neutrino hierarchy is not the normal one. In this case, in fact, there are no ideas on how to sense this region of the parameter space.

\subsection{Germanium diodes}

The Heidelberg-Moskow (HM) experiment operated at the Laboratori Nazionali del Gran Sasso (LNGS) in Italy five high purity Germanium diodes (HPGe). The detectors were enriched in $86 \%$ ${ }^{76} \mathrm{Ge}$ and featured a high energy resolution $(\sim 4 \mathrm{keV}$ FWHM) and very low background $(0.12$ counts $/ \mathrm{keV} / \mathrm{kg} / \mathrm{y}$ ). A statistics of $10 \mathrm{~kg} \times 3.5 \mathrm{y}$ was collected, and no evidence of $0 v \mathrm{DBD}$ was found. The half-life limit of ${ }^{76} \mathrm{Ge}$ was set to $T_{1 / 2}^{0 v}>1.9 \times 10^{25} \mathrm{y}$ at $90 \%$ C.L [10]. In 2001 a subset (KHDK) of the collaboration claimed evidence for a $0 v \mathrm{DBD}$ signal with $T_{1 / 2}^{0 v}=2.23_{-0.31}^{+0.44} \times 10^{25} \mathrm{y}[11,12]$ (Fig. 2 left). The result, based on a re-analysis of the HM data, has raised criticisms because the understanding of the background in the region of the peak is not clear, but cannot be dismissed out of hand.

GERDA [13] is the evolution of the Heidelberg-Moscow experiment. Since the main source of background in HM was due to $\gamma$ particles, the detectors, which were operated in liquid nitrogen, are now operated in a liquid argon bath, with the aim of using it as active veto. Another tool to reject the background is provided by the pulse shape discrimination, already used in the Heidelberg-Moscow experiment with remarkable results. 

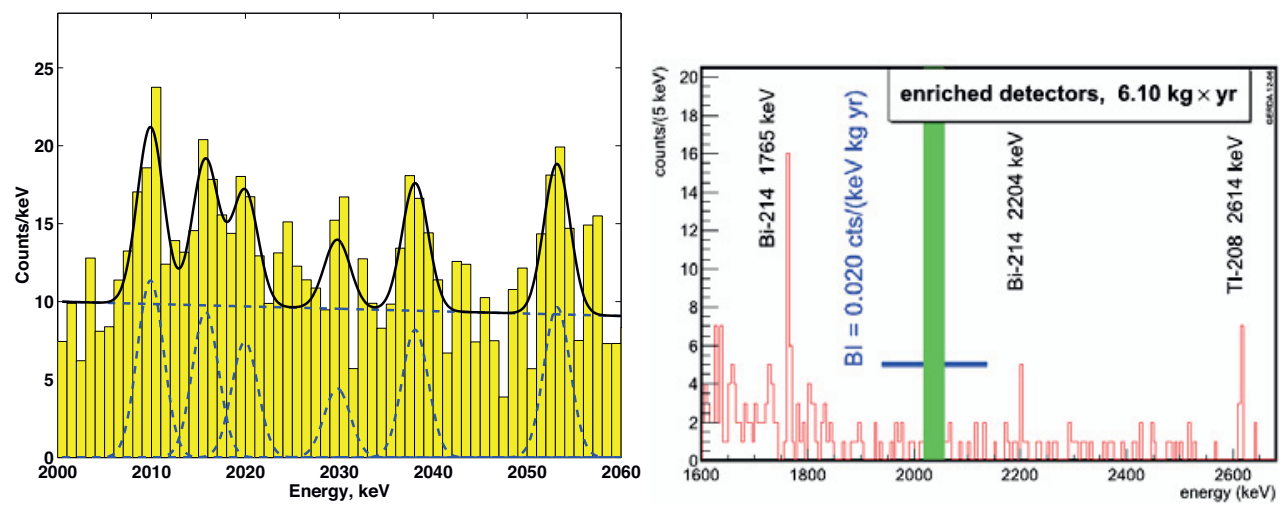

Figure 2. Left: The controversial claim published in [11] using data from the Heidelberg-Moskow experiment; the peak at $2039 \mathrm{keV}$ is attributed to $0 v \mathrm{DBD}$. Right: The current spectrum of the GERDA experiment [15]; the region around the Q-value is blinded.

The first phase [14] started in June 2011 with $14.6 \mathrm{~kg}$ of ${ }^{76} \mathrm{Ge}$ enriched detectors and $7.6 \mathrm{~kg}$ of natural Ge diodes. The comparison of the spectra of the enriched and non-enriched detectors will allow to determine whether a peak in the region of interest is effectively due to $0 v \mathrm{DBD}$ or to an unrecognized background. From the first data (Fig. 2 right), the background in the $0 v \mathrm{DBD}$ region is 0.02 counts $/ \mathrm{keV} / \mathrm{kg} / \mathrm{y}$, thus allowing the check of the KHDK claim in 1.5 years of data taking.

The second phase foresees the deployment of around $35 \mathrm{~kg}$ of ${ }^{76} \mathrm{Ge}$ enriched $\mathrm{B}$ (road)E(nergy) Ge detectors, which will allow to further suppress the $\gamma$ background. $\gamma$ 's with energies close to the Q-value of ${ }^{76} \mathrm{Ge}(2039 \mathrm{keV})$ give rise to multiple-site interactions, while $\beta$ 's give rise to single site interactions. In BEGe diodes the electric field is not uniform in the depletion zone. While the signal of $\beta$ 's will have a concentrated structure in time, the signal from $\gamma$ 's will be broader, because of the different paths of the charge from the sites to the contact. This enhanced pulse shape capability, together with the active veto, should allow to reach a background of the order of 1 count $/ \mathrm{keV} / \mathrm{ton} / \mathrm{y}$ and a $5 y$ sensitivity of $\sim 2.5 \times 10^{26} \mathrm{y}$.

Majorana $[16,17]$ is another experiments using HPGe detectors. The detectors will be installed in several separate cryostats that will be hosted in the Deep Underground Science and Engineering Laboratories (Dusel, 4,200 m w.e.) in South Dakota. The MAJORANA collaboration plan to reach a background level smaller than $10^{-3}$ counts $/ \mathrm{keV} / \mathrm{kg} /$ year in the $0 v \mathrm{DBD}$ region. The expected sensitivity after ten years of data taking is $6 \cdot 10^{27} \mathrm{y}\left(m_{\beta \beta} 0.01-0.04 \mathrm{eV}\right)$. A demonstrator experiment with a total mass of $40 \mathrm{~kg}$ will be operated starting from 2013. The ultimate goal of this experiment is to deploy, together with the GERDA collaboration, an array of HPGe detectors enriched at $86 \%$ in ${ }^{76} \mathrm{Ge}$ for a total mass of the order of one ton.

\section{Bolometers}

Bolometers are sensitive calorimeters, operated at a few $\mathrm{mK}$, that measure the rise in temperature due to the energy deposited by particle interactions.

CUORICINO [18] was an array of $62 \mathrm{TeO}_{2}$ bolometers installed at LNGS. It operated a $\mathrm{TeO}_{2}$ mass of $40.7 \mathrm{~kg}$, that corresponds to $11 \mathrm{~kg}$ of ${ }^{130} \mathrm{Te}$. The experiment took data in the period 20032008. A very low background, comparable with the HM experiment, was achieved together with an 
average resolution of $\sim 7 \mathrm{keV}$ FWHM. With a total statistics of $19.7 \mathrm{~kg} \times \mathrm{y}$ CUORICINO set a limit of $T_{1 / 2}^{0 v}>2.8 \times 10^{24} \mathrm{y}$, corresponding to $m_{\beta \beta}<300-700 \mathrm{meV}$. The large uncertainty, which is introduced by the nuclear matrix elements, did not allow to disprove the KHDK claim.

CUORE [19], the successor of CUORICINO, will consist in an array of $988 \mathrm{TeO}_{2}$ bolometers arranged in a cylindrical configuration of 19 towers containing 52 crystals each $(750 \mathrm{~g})$, for a total mass of $741 \mathrm{~kg}$. Each of these towers is a CUORICINO-like detector consisting in 13 modules, 4 detectors each. The expected energy resolution is $\sim 5 \mathrm{keV} \mathrm{FWHM}$ at the $0 v \mathrm{DBD}$ transition energy ( $~$ $2.527 \mathrm{KeV}$ ). A background level of $\sim 0.01 \mathrm{c} / \mathrm{keV} / \mathrm{kg} / \mathrm{y}$ is expected by extrapolating the CUORICINO background results and the dedicated CUORE R\&D measurements. The expected 5y sensitivity is $1.6 \times 10^{26}$ y [20] $\left(m_{\beta \beta}<40-96 \mathrm{meV}\right)$. CUORE is fully funded and presently under construction at LNGS at a relatively low cost thanks to the high natural abundance of ${ }^{130} \mathrm{Te}$. Setup completion is expected in 2014.

The Q-value of ${ }^{130} \mathrm{Te}$ is between the Compton edge and photo-electric peak of the $2615 \mathrm{keV}$ line of ${ }^{208} \mathrm{Tl}$, therefore the $\gamma$ background is much smaller than in Ge detectors. However the CUORICINO data indicated that the background of $\mathrm{TeO}_{2}$ bolometers is dominated by $\alpha$ particles which deliver part of their energy in the materials close to the detectors and the rest in the detector itself. Since $\alpha$ particles of few MeV have short interaction lengths, of the order of few tens of $\mu \mathrm{m}$, the diodes of GERDA do not experience this background, since their surface is insensitive to energy depositions.

However, thanks to the bolometer's versatility, alternative options with respect to $\mathrm{TeO}_{2}$ are also possible. Beautiful results have been obtained so far with scintillating bolometers [21-26] which could allow to study in the future new $0 v \mathrm{DBD}$ active isotopes with improved sensitivity. LUCIFER [27] will consist in an array of scintillating bolometers operated at $10-20 \mathrm{mK}$. The basic idea consists in developing a scintillating bolometer containing a candidate isotope with $Q>2615 \mathrm{keV}$, therefore outside the natural radioactivity range. The background source in this energy region is dominated by surface $\alpha$ particles. Because of the different light yield for the same energy deposition, $\alpha$ particles and electrons can be separated measuring simultaneously the energy they deliver in the form of heat and the scintillation light they produce. Thanks to this discrimination, it is possible to approach a zero background with exposure of the order of tons.year. The proof of principle with $\sim 10 \mathrm{~kg}$ of enriched $\mathrm{Se}$, embedded in $\mathrm{ZnSe}$ scintillating crystals is foreseen in 2015. Another very promising compound that will be investigated is $\mathrm{ZnMoO}_{4}$ [23-26]. LUCIFER is in the R\&D phase, and can be considered as a demonstrator for a possible upgrade of CUORE, with however a considerable sensitivity by itself.

Unfortunately $\mathrm{TeO}_{2}$ bolometers do not scintillate. An alternative option, that matches the low cost and high performance of $\mathrm{TeO}_{2}$ detectors with the discrimination capability offered by the light emission, is the Čerenkov light detection. At this energy, in fact, electrons are above the Čerenkov threshold while $\alpha$ particles do not. Promising results have recently been obtained [28].

\section{Lliquid scintillators}

EXO [29] is a liquid Xenon (LXe) TPC detector with an energy resolution of 3.8\% FWHM thanks to the simultaneous measurement of ionization and scintillation light. The set-up provides event position and topology, thus allowing the rejection of the multi-site events induced by the $\gamma$ background. A small prototype experiment with a Xe mass of $200 \mathrm{~kg}\left(80 \%{ }^{136} \mathrm{Xe}\right)$, is taking data at the WIPP facility from Spring 2011. EXO-200 measured for the first time the ${ }^{136} \mathrm{Xe} 2 v$ DBD half-life [30] and, with a background of 1.5 counts $/ \mathrm{keV} / \mathrm{ton} / \mathrm{y}$ in the $0 v \mathrm{DBD}$ region, was able to set a limit on $m_{\beta \beta}$ in the range 140-380 meV [31]. This is the most competitive limit in the field and rules out almost completely the KHDK claim (Fig. 3).

The full scale experiment foresees $\sim 1-10$ tons of isotopically enriched $\left(85 \%\right.$ in $\left.{ }^{136} \mathrm{Xe}\right) \mathrm{Xe}$. To completely remove the background, the collaboration is developing a technique to rescue and tag the 
charged $\mathrm{Ba}$ isotope produced in the decay $\left({ }^{136} \mathrm{Xe} \rightarrow{ }^{136} \mathrm{Ba}^{++}+2 e^{-}\right)$. The technical feasibility of such an ambitious project requires a hard and still ongoing $R \& D$ phase.
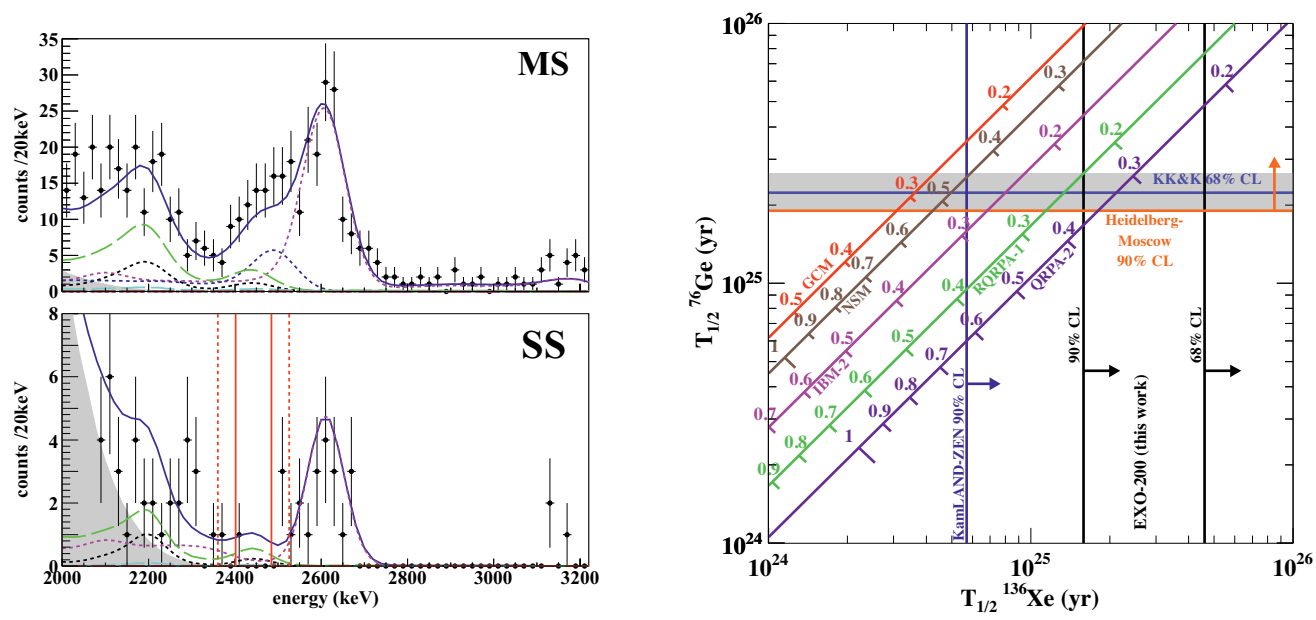

Figure 3. Left: Energy spectrum of EXO-200 around the ${ }^{136} \mathrm{Xe}$ Q-value in multi-site and single-site events. 1 event is observed in a $\pm 1 \sigma$ interval, while 5 are observed in a $\pm 2 \sigma$ interval, which is consistent with the background expectations. Right: Half-life of ${ }^{76} \mathrm{Ge}$ versus half-life of ${ }^{136} \mathrm{Xe}$ for different evaluations of the nuclear matrix elements. The recent limits from EXO-200 exclude almost completely the KHDK claim. Pictures taken from [31].

Both SNO+ [32] and KAMLAND-Zen [33] experiments use a different strategy: the $0 v$ DBD active isotope is dispersed in a large mass of low-radioactive scintillator. They aim is to compensate the modest energy resolution of this kind of detectors with a large statistics.

$\mathrm{SNO}+$ is pursuing the goal of studying ${ }^{150} \mathrm{Nd}$ with $50-500 \mathrm{~kg}$ of isotopically enriched Neodimium loaded liquid scintillator depending on the results of the currently ongoing R\&D program. This search will depend critically on the possibility to enrich $\mathrm{Nd}$ in ${ }^{150} \mathrm{Nd}$. Another issue concerns the ${ }^{150} \mathrm{Nd}$ nuclear matrix elements, whose calculation is made difficult by nucleus deformation, which could lead to an important suppression. The present plan is to use $0.1 \% \mathrm{w} / \mathrm{w}$ natural Nd-loaded liquid scintillator in 1000 tons, providing a source of $56 \mathrm{~kg}{ }^{150} \mathrm{Nd}$, which should guarantee an energy resolution of $\sim 6 \%$ FWHM and a sensitivity of $100-200 \mathrm{meV}$ to $m_{\beta \beta}$ in 3 years. Data taking is foreseen in 2015 .

A similar approach is pursued by KamLAND-ZEN [33] but using ${ }^{136} \mathrm{Xe}$ as active isotope. The first phase started in Septmber 2011. A mini-balloon with $330 \mathrm{~kg}$ of isotope $90 \%$ enriched in ${ }^{136} \mathrm{Xe}$ dissolved in liquid scintillator was immersed in the KamLand main vessel. An unexpected background (probably due to cosmogenic activation or Fukushima fall-out) showed up in the $0 v$ DBDregion thus limiting the experimental sensitivity. An $R \& D$ effort is on going to mitigate this background and proceed to the second phase with 1 ton of Xenon enriched to $90 \%$ in ${ }^{136} \mathrm{Xe}$.

\section{Other techniques}

NEXT [34] is a proposed $100 \mathrm{~kg}$ high-pressure gaseous-xenon TPC, to be located in CANFRANC, Spain. The extension to 1 ton is technically possible. Clear two-track signature is achievable, thanks to 
the use of gaseous rather than liquid Xe. The estimated energy resolution is of the order of $1 \%$ FWHM, achieved thanks to the electroluminescence signal associated to the ionization electrons produced by the Double Beta Decay events. The experiment is in the R\&D phase. The first prototype NEXT- 1 is completed. A mid-scale experiment NEXT-10 (10 kg isotope) is under preparation [35].

COBRA [36] is a proposed array of ${ }^{116} \mathrm{Cd}$-enriched CdZnTe semiconductor detectors at room temperature. Nine isotopes are under test in principle, but ${ }^{116} \mathrm{Cd}$ is the only competing candidate. The final aim of the project is to deploy $117 \mathrm{~kg}$ of ${ }^{116} \mathrm{Cd}$ with high granularity. Small scale prototypes have been realized at LNGS. The proved energy resolution is $1.9 \%$ FWHM. The project is in R\&D phase. Results on pixelization [37] shows that the COBRA approach may allow an excellent tracking capability (solid state TPC).

NEMO3 [38] was an inhomogeneous detector in which the electrons emitted by the sources cross a magnetized tracking volume instrumented with Geiger cells and deliver their energy to a plastic scintillating calorimeter. The detector was operated at Laboratoire Souterrain Modan, in France. The big advantage of the technique relied on the possibility to study many candidates simultaneously and to access single electron informations which allow $2 v$ DBD decay to be reconstructed with no other background. On the other hand, the poor energy resolution of about 12\% FWHM and the limited amount of $0 v$ DBD mass source (due to the unavoidable foil structure) limited the $0 v$ DBD sensitvity.

Super-NEMO [39] is an extension of the NEMO3 concept, scaled in order to accommodate $\sim 100$ $\mathrm{kg}$ of ${ }^{82} \mathrm{Se}$ foils spread among 20 detector modules. The proposed geometry is planar. The energy resolution will be improved from 12\% FWHM to 7\% FWHM in order to reduce the background due to $2 v$ DBD. The signal detection efficiency will also improve from $8 \%$ to $40 \%$. The detector modules will have an active water shield to further reduce cosmic ray backgrounds. A demonstrator (single module) is presently fully funded to be completed in 2012 with a test run in the current NEMO3 site.

\section{References}

[1] M. Goeppert-Mayer, Phys. Rev. 48, 512 (1935)

[2] W.H. Furry, Physical Review 56, 1184 (1939)

[3] E. Majorana, Nuovo Cimento 14, 171 (1937)

[4] S. Bilenky, C. Giunti, Mod.Phys.Lett. A27, 1230015 (2012), 1203.5250v2

[5] V. Rodin, J.Phys.Conf.Ser. 375, 042025 (2012)

[6] B. Schwingenheuer, J. Phys. Conf. Ser. 375, 042007 (2012)

[7] M. Redshaw, B.J. Mount, E.G. Myers, F.T. Avignone, Phys. Rev. Lett. 102, 212502 (2009)

[8] N. Scielzo et al., Physical Review C 80 (2009)

[9] S. Rahaman, V. Elomaa, T. Eronen, J. Hakala, A. Jokinen et al., Phys.Lett. B703, 412 (2011)

[10] H.V. Klapdor-Kleingrothaus et al., Eur. Phys. J. A12, 147 (2001), hep-ph/0103062

[11] H.V. Klapdor-Kleingrothaus, I.V. Krivosheina, A. Dietz, O. Chkvorets, Physics Letters B 586, 198 (2004), hep-ph/0404088

[12] H.V. Klapdor-Kleingrothaus, I.V. Krivosheina, Mod. Phys. Lett. A21, 1547 (2006)

[13] I. Abt et al. (2004), hep-ex/0404039

[14] K. Knopfle (GERDA Collaboration), AIP Conf.Proc. 1441, 483 (2012)

[15] P. Grabmayr (GERDA Collaboration), to appear in the proceedings of XXV International Conference on Neutrino Physics and Astrophysics (2012)

[16] R. Gaitskell et al. (Majorana) (2003), nucl-ex/0311013

[17] V.E. Guiseppe et al. (Majorana) (2008), 0811.2446

[18] E. Andreotti et al., Astropart.Phys. 34, 822 (2011), 1012.3266 
[19] C. Arnaboldi et al., Nucl. Instrum. Meth. A. 518, 775 (2004), hep-ex/0212053v1

[20] F. Alessandria, E. Andreotti, R. Ardito, C. Arnaboldi, I. Avignone, F.T. et al. (2011), 1109. 0494

[21] C. Arnaboldi et al., Astropart.Phys. 34, 344 (2011), 1006. 2721

[22] C. Arnaboldi et al., Astropart.Phys. 34, 143 (2010), 1005. 1239

[23] C. Arnaboldi et al., Astropart. Phys. 34, 797 (2011), 1011.5415

[24] J. Beeman et al., Astroparticle Physics 35, 813 (2012)

[25] J. Beeman et al. (2012), 1207.0433

[26] J. Beeman, F. Bellini, C. Brofferio, L. Cardani, N. Casali et al. (2012), 1207.0433

[27] F. Ferroni, Nuovo Cimento C 033, 27 (2010)

[28] J. Beeman et al. (2011), submitted to Astropart. Phys., 1106.6286

[29] M. Auger et al., J. Instrum. 7, P05010 (2012)

[30] N. Ackerman et al. (EXO-200 Collaboration), Phys.Rev.Lett. 107, 212501 (2011), 1108. 4193

[31] M. Auger et al. (EXO Collaboration), Phys.Rev.Lett. 109, 032505 (2012), 1205.5608

[32] J. Hartnell (SNO+ Collaboration) (2012), arXiv: 1201.6169

[33] A. Gando et al. (KamLAND-Zen Collaboration), Phys.Rev. C85, 045504 (2012), 1201.4664

[34] V. Alvarez (NEXT Collaboration) (2011), 1106.3630

[35] R. Santorelli (NEXT Collaboration), AIP Conf.Proc. 1441, 486 (2012)

[36] K. Zuber, Physics Letters B 519, 1 (2001), nucl-ex/0105018v1, nucl-ex/0105018v1

[37] T. Bloxham et al. (COBRA), Physical Review C 76, 025501 (2007), 0707.2756v1 [nucl-ex], Q707.2756v1 [nucl-ex]

[38] L. Simard (NEMO-3 Collaboration), J.Phys.Conf.Ser. 375, 042011 (2012)

[39] F. Piquemal (NEMO), Phys. Atom. Nucl. 69, 2096 (2006) 